\title{
Does Comorbid Anxiety Counteract Emotion Recognition Deficits in Conduct Disorder?
}

Roxanna M.L. Short ${ }^{1}$, Edmund J.S. Sonuga-Barke ${ }^{1,2}$, Wendy J. Adams ${ }^{1}$, Graeme Fairchild ${ }^{1}$

${ }^{1}$ Academic Unit of Psychology, University of Southampton, Southampton, UK

${ }^{2}$ Department of Experimental, Clinical and Health Psychology, Ghent University, Ghent, Belgium

Total word count: 6778

Abstract word count: 242

Short title: Emotion recognition in conduct disorder and anxiety disorders

Declaration of potential conflicts of interest:

Sonuga-Barke: fees for speaking, consultancy, research funding and conference support from Shire Pharmaceuticals; speaker fees from Janssen Cilag, Medice \& Qbtech; book royalties from OUP and Jessica Kingsley and; consultancy from Neurotech solutions.

The other authors declare no conflicts of interest. 
Abstract

Background: Previous research has reported altered emotion recognition in both conduct disorder (CD) and anxiety disorders (ADs) - but these effects are of different kinds. Adolescents with CD often show a generalised pattern of deficits, while those with ADs show hypersensitivity to specific negative emotions. Although these conditions often co-occur, little is known regarding emotion recognition performance in comorbid CD+ADs. Here we test the hypothesis that in the comorbid case, anxietyrelated emotion hypersensitivity counteracts the emotion recognition deficits typically observed in $C D$.

Method: We compared facial emotion recognition across four groups of adolescents aged 12-18 years: those with $C D$ alone $(n=28)$, ADs alone $(n=23)$, co-occurring $C D+A D s(n=20)$ and typicallydeveloping controls $(n=28)$. The emotion recognition task we used systematically manipulated the emotional intensity of facial expressions as well as fixation location (eye, nose or mouth region).

Results: CD was associated with a generalised impairment in emotion recognition, however this may have been modulated by group differences in IQ. AD was associated with increased sensitivity to low intensity happiness, disgust and sadness. In general, the comorbid CD+ADs group performed similarly to typically-developing controls.

Conclusions: Although CD alone was associated with emotion recognition impairments, ADs and comorbid $C D+A D s$ were associated with normal or enhanced emotion recognition performance. The presence of comorbid ADs appeared to counteract the effects of $C D$, suggesting a potentially protective role, although future research should address the contribution of IQ and gender to these effects.

Keywords: conduct disorder, anxiety disorder, callous-unemotional traits, comorbidity, emotion recognition, response biases, social information processing 
Introduction

Conduct disorder (CD) is a common condition that emerges in childhood or adolescence, and is characterised by rule-breaking, aggression and delinquency (American Psychiatric Association, 2013). CD entails a considerable economic and social burden (Scott, Henderson, Knapp, \& Maughan, 2001) and is linked to unfavourable adult outcomes such as antisocial personality disorder and persistent criminality (Robins, 1978).

CD frequently co-occurs with other disorders; see Angold, Costello, and Erkanli (1999) for a review. For example, there is an elevated rate of anxiety disorders (ADs) in individuals with CD (Greene et al., 2002; Polier, Vloet, Herpertz-Dahlmann, Laurens, \& Hodgins, 2012). The reason for this cooccurrence is unknown (e.g. Lahey, Loeber, Burke, Rathouz, \& McBurnett, 2002) although some argue that there may be a specific anxiety-mediated developmental pathway to CD (e.g. Frick, Lilienfeld, Ellis, Loney, \& Silverthorn, 1999), linked to emotional dysregulation/reactivity (Frick \& Morris, 2004). This may lead to hyper-sensitivity to perceived threat and reactive aggression. The prognosis for individuals with both $C D$ and $A D s$ is uncertain. Some studies report a more benign outcome for comorbid individuals (e.g. Walker et al., 1991), while others suggest that anxiety exacerbates the effects of CD (lalongo, Edelsohn, Werthamer-Larsson, Crockett, \& Kellam, 1996; Kendall, Brady, \& Verduin, 2001; Sourander et al., 2007).

Given its prevalence, we need a better understanding of the mechanisms underpinning the comorbidity between $\mathrm{ADs}$ and $\mathrm{CD}$. One mechanism that may be important relates to the way other people's expressions of emotion are processed: deficits in emotion recognition have been reported in adolescents with CD (e.g. Fairchild, Van Goozen, Calder, Stollery, \& Goodyer, 2009) and ADs (e.g. Leist \& Dadds, 2009). Consistent with the idea that they are insensitive to others' distress cues, a meta-analysis found specific deficits in fear recognition in individuals displaying antisocial behaviour (Marsh \& Blair, 2008). CD is also associated with deficits in a broader set of emotions including anger, disgust and surprise (Fairchild, Stobbe, van Goozen, Calder, \& Goodyer, 2010; Fairchild et al., 2009; Sully, Sonuga-Barke, \& Fairchild, 2015), although see Pajer, Leininger, and Gardner (2010) for a null finding. Adolescents with psychopathic/CU traits, which are often associated with $C D$, show impaired fear and sadness recognition (Blair \& Coles, 2000; Dadds et al., 2006). However, given the extensive overlap between $\mathrm{CD}$ and $\mathrm{CU}$ traits, isolating their unique impact on emotion recognition is difficult. 
Within CD samples, psychopathic traits have been associated with deficits in sadness (Fairchild et al., 2010; Fairchild et al., 2009) and fear recognition (Fairchild et al., 2009). It has been suggested that the fear recognition deficits seen in those with CU traits may be due to impaired attention to the eyeregion of the face: Dadds et al. (2006) found that fear recognition in high CU trait adolescents normalised when they were instructed to fixate the eye-region of the faces, and in a follow-up study using eye-tracking, Dadds, El Masry, Wimalaweera, and Guastella (2008) found that adolescents with psychopathic traits showed reduced eye-fixation in an emotion recognition task.

Altered facial emotion recognition has also been reported in ADs, although findings are inconsistent, with different studies reporting normal (Guyer et al., 2007; Manassis \& Young, 2000; McClure, Pope, Hoberman, Pine, \& Leibenluft, 2003; Melfsen \& Florin, 2002), enhanced (Jarros et al., 2012; ReebSutherland et al., 2015), and inferior performance in those with ADs relative to controls (Battaglia et al., 2010; Simonian, Beidel, Turner, Berkes, \& Long, 2001). Individuals with ADs may also be hypersensitive to stimuli conveying threat, however this typically manifests as an attentional bias towards threat (e.g. Bar-Haim, Lamy, Pergamin, Bakermans-Kranenburg, \& van IJzendoorn, 2007). Unfortunately, comorbidity has rarely been considered in these studies and some of the effects attributed to ADs may have been explained by the presence of other disorders (e.g., unmeasured $\mathrm{CD}$ ). For example, a study on emotion recognition in depressed children with comorbid CD found that comorbid individuals did not display the same biases as their depressed counterparts (Schepman, Taylor, Collishaw, \& Fombonne, 2012), suggesting that comorbid CD may attenuate the effect of depression on the negative evaluation of low-intensity facial expressions.

The present study is the first to examine facial emotion recognition in adolescents with both $C D+A D s$, those with pure versions of each condition, and typically-developing controls. We predict that CD will be associated with a reduced ability to discriminate between emotions in general, which is especially pronounced for distress cues (fear and sadness). In contrast, we hypothesise that individuals with ADs will show hypersensitivity to threat-related expressions (anger and fear). Our hypothesis regarding comorbid $C D+A D s$ is somewhat more speculative and is based on the idea that the enhanced sensitivity in ADs may counteract the deficits observed in CD, producing a "protective" effect with comorbid $C D+A D$ s individuals performing similarly to controls. 
Our study addresses a number of methodological limitations present in previous studies: emotional faces were morphed at different intensities, allowing evaluation of subtle deficits and biases in the appraisal of emotional expressions (e.g. Schönenberg \& Jusyte, 2014). Classification of neutral or low-intensity facial expressions may provide insights into attributional biases (Crick \& Dodge, 1994). We additionally controlled fixation location, to assess whether this modulates emotion recognition, thereby testing previous assertions that fear recognition deficits related to $\mathrm{CU}$ traits are driven by impaired attention to the eye-region of the face (Dadds et al., 2006). Finally, we assessed both sensitivity (i.e. the ability to discriminate between stimuli) and bias (i.e. the tendency to make a particular response); response accuracy is dependent on both factors.

Methods

Participants

We recruited 99 adolescents aged 12 to 18 years $(M=16.6, S D=1.5,38.5 \%$ girls) from schools, colleges, Youth Offending Teams and Pupil Referral Units. All participants (and parents/carers, if below age 16) provided informed consent. Inclusion criteria were $I Q \geq 75$ (measured with the vocabulary and matrix reasoning subtests of the Wechsler Abbreviated Scale of Intelligence; Wechsler, 1999), and being free of pervasive developmental disorder or psychosis. All participants were assessed for DSM-IV criteria for CD, ADHD, generalised anxiety disorder (GAD), major depressive disorder (MDD), social phobia, specific phobia, panic disorder, obsessive-compulsive disorder (OCD), alcohol or substance abuse, posttraumatic stress disorder (PTSD), and oppositional defiant disorder (ODD), using a semi-structured clinical interview: the Kiddie Schedule for Affective Disorders and Schizophrenia-Present and Lifetime version (K-SADS-PL; Kaufman et al., 1997). Of the 99 participants, 46 met criteria for current CD and/or ODD. Of this group, 20 additionally met criteria for an AD. 23 participants met AD criteria but had no current or lifetime diagnosis of CD/ODD. Comorbidity with other disorders was common (see Table S1 in Supplementary Materials). Twentynine healthy controls screened negative for any disorder.

\section{Procedure}

After the clinical interviews, participants completed a range of laboratory tasks and questionnaires during a 3.5 hour testing session at the University. 
Measures

Clinical assessment: The K-SADS-PL (Kaufman et al., 1997) was administered by trained interviewers with participants and parents (interviewed separately). A symptom was considered present if endorsed by either informant. Individuals were allocated to the CD group if they met the criteria for $C D$ ( $\geq 3 C D$ symptoms currently present), or if they met full criteria for ODD with 1-2 CD symptoms (three participants)

CU traits: The self-report Inventory of Callous-Unemotional Traits (ICU; Frick, 2004) is a 24-item questionnaire focusing on affective components of psychopathy (Cronbach's alpha $=0.84$ ).

Trait anxiety: The trait subscale of the State-Trait Anxiety Inventory (STAI; Spielberger, Gorsuch, Lushene, Vagg, \& Jacobs, 1983), a 20 -item self-report questionnaire, was used to assess anxiety (Cronbach's alpha=0.93).

Current depressive symptoms: were assessed using the Hospital Anxiety and Depression Scale (HADS; Zigmond \& Snaith, 1983); Cronbach's alpha=0.73.

Social disadvantage: The 18-item Neighbourhood Environment Scale (NES; Crum, Lillie-Blanton, \& Anthony, 1996) was used as a proxy-measure of socioeconomic status (Cronbach's alpha=0.81).

Facial identity recognition: The Benton Facial Recognition Test (BFRT; Benton, Hamsher, Varney, \& Spreen, 1994) was used to assess participants' basic facial recognition skills. Participants were required to identify target faces from an array of unfamiliar faces.

Facial emotion recognition: We assessed anger, fear, happiness, sadness and disgust recognition using a five-alternative-forced-choice task. Face stimuli were selected from the NimStim MacArthur Network Face Stimuli Set (Research Network on Early Experience and Brain Development, http://www.macbrain.org/resources.htm, Tottenham et al., 2009). Images of the actors were combined to create one male and one female face for each emotional and neutral expression, using a morphing algorithm implemented in MATLAB® (The Mathworks, 2012) (see Adams, Gray, Garner, \& Graf, 2010). Faces were converted to greyscale and matched on contrast and luminance. Averaged emotional faces were combined with averaged neutral faces in varying proportions to produce expressions of different intensities. Fearful, disgusted, angry and sad faces were created with 
$18.75 \%, 37.50 \%, 56.25 \%$ and $75.00 \%$ intensities. Pilot data indicated that happy faces were more easily discriminated than the other emotions. Thus, to avoid ceiling effects, happy faces were created with $12.5 \%, 25.0 \%, 37.5 \%$ and $50 \%$ intensities (see Figure 1, top panel). In total, 42 images were used in the task ( 5 emotions $\times 4$ intensities $\times 2$ genders, plus 1 male and 1 female neutral face). An oval mask was used to remove non-facial features (e.g. hair). Stimuli were presented on a monitor at a viewing distance of $65 \mathrm{~cm}$ and subtended $7.8 \times 11.6$ degrees of visual angle.

To initiate trials, participants used the mouse to click on a central fixation cross. A face was then immediately presented at one of three vertical locations: the fixation position corresponded to the eyes, nose or mouth. After $250 \mathrm{~ms}$ a mask was shown; this presentation time prevented multiple fixations (Rayner, 1998). The participant used the mouse to identify the facial emotion by selecting one emotion label (see Figure 1, bottom panel). Each emotional face was presented three times, and each neutral face was presented six times, in each of the three fixation positions (396 trials in total). Trials were presented in a random order in four blocks of 99 trials.

[Figure 1 about here]

Data preparation

Following a signal detection approach, d-Prime $\left(d^{\prime}\right)$ scores were calculated for each emotion at each intensity and fixation position (averaged across stimulus gender) from the corresponding correct identification (hit) rates and misidentification (false alarm) rates. False alarm rates were calculated by averaging the misidentifications for an emotion at each intensity level and fixation position. Hit- and false alarm- rates of 0 or 1 (resulting in an infinite $d^{\prime}$ ) were converted to $1 / 2 \mathrm{~N}$ and $1-1 / 2 \mathrm{~N}$, respectively, where $\mathrm{N}$ was the number of trials for that condition (Miller, 1996).

Response bias was quantified by the proportion of trials in which neutral faces were misclassified as each emotion.

Data analysis

First, groups were compared in demographic and clinical characteristics using one-way ANOVAs and Bonferroni-corrected post-hoc tests. Chi-Squared tests were used to compare groups on categorical variables. Second, we performed preliminary analyses to test where morph strength and fixation 
position interacted with either CD or AD in terms of emotion recognition. Where this was not the case, these were dropped from further analysis to promote easier interpretation of the core results. In these analyses, $d$ ' was the dependent variable in a 5 (emotion) $\times 4$ (morph strength) $\times 3$ (fixation position) $x$ 2 (CD: present, $C D+/ a b s e n t, C D-) \times 2$ (AD: present, AD+/absent, AD-) mixed-design ANOVA. Where fixation position did not interact with $\mathrm{CD}$ or $\mathrm{AD}$ status, $d$ scores were re-calculated and entered into a 5 (emotion) $\times 4$ (morph strength) $\times 2$ (CD) $\times 2$ (AD) mixed ANOVA. Third, separate two-way ANOVAs investigated the effects of $C D$ (present or absent) and $A D$ (present or absent) on the misclassification of neutral faces, for each emotion. Bonferroni-corrected post-hoc simple effects analyses were conducted to explore any resulting interaction effects. Effect sizes for the simple effects analyses are reported as Pearson's $r$ (small $\geqslant 0.1$, medium $\geqslant 0.3$, large $\geqslant 0.5$; Cohen, 1992). Fourth, to examine the effects of $\mathrm{CU}$ traits within the CD groups (i.e., across the $C D$ and comorbid groups), we conducted a 5 (emotion) $\times 4$ (morph strength) $\times 3$ (fixation position) $\times 2$ (AD) $\times 2$ (CU: high, CU+/low, CU-) repeated-measures ANOVA. Given the non-linear relationship between CU traits and emotion recognition, individuals were classified as high/low CU traits on the basis of a median split (CU+ $\geq 30$ on the ICU). Hierarchical regression analyses were used to examine the influence of confounding variables that differed between groups and were significantly correlated with outcome variables associated with $\mathrm{CD}$ or $\mathrm{AD}$.

Results

Participant characteristics (see Table 1).

The AD group had more fear-related ADs, such as Panic Disorder, Specific and Social Phobias $\left(\mathrm{X}^{2}=\right.$ 11.29, $p<0.01$; see Table S1). The comorbid group had more worry-based ADs, such as GAD and $\operatorname{OCD}\left(x^{2}=3.84, p=0.05\right)$. The $C D$ and comorbid groups had similar rates of ADHD $\left(X^{2}=0.72, p=\right.$ 0.39). The three clinical groups had similarly elevated rates of $\operatorname{MDD}\left(x^{2}=2.54, p=0.28\right)$.

The $C D$ and comorbid groups had significantly more $C D$ symptoms and elevated $C U$ traits than the $A D$ and control groups (all $p<0.01$ ). The $C D$ and comorbid groups did not differ from each other in $C U$ traits $(p=0.68)$. The comorbid and AD groups were elevated in trait anxiety relative to the $C D$ and control groups (all $p<0.05$ ). The comorbid and CD groups reported more depressive symptoms than controls (both $p<0.01$ ). Although the groups were matched for age and socioeconomic status, the 
CD group had a lower IQ than the control group $(p<0.01)$. In addition, the proportion of females was higher in the AD group than the other groups $\left(x^{2}=24.04, p<0.01\right)$.

[Table 1 about here]

Preliminary analyses: There was a main effect of morph strength: as expected, more intense emotional expressions were easier to recognise $(F(2,165)=474.67, p<0.01)$. There was also an effect of fixation position $(F(2,190)=20.00, p<0.01)$, and an emotion $\mathrm{x}$ fixation interaction $(F=3.61$, $p<0.01)$. Mouth fixation resulted in the poorest performance for all emotions, except for disgust where there were no differences. For the other emotions, central/nose fixation resulted in the best performance, except for fear where eye fixation led to enhanced performance (see Figure S1). There were main effects of emotion $(F(3,257)=110.22, p<0.01)$. Happiness was the easiest to recognise. Fear and sadness were more easily recognised than anger and disgust, but only for the two lowest morph strengths (all $p<0.01$ ). At the highest morph strength, anger and fear were more easily recognised than sadness and disgust (all $p<0.01$ ), and sadness was more easily recognised than disgust $(p<0.01)$. Morph strength interacted with $C D$ and $A D$ and was therefore retained in the analyses (see below).

Effects of $C D$ and $A D$ : Individuals with $C D$ performed worse than their non-CD counterparts across emotions $(F(1,95)=4.97, p=0.03, r=0.22$; see Figure 2). This effect was strongest for the highest morph strengths, as indicated by a CD x morph strength interaction $(F(2,165)=4.23, p=0.02$; see Figure S2). There was no main effect of $\mathrm{AD}$ status $(\mathrm{F}(1,95)=1.17, p=0.28, r=0.11)$ on emotion discrimination. Although the interaction between $C D$ and $A D$ status was non-significant $(p=0.27)$, the CD group tended to show larger deficits than the comorbid group $(F(1,95)=2.87, p=0.09, r=0.17)$. In fact, neither the AD nor the comorbid group differed from controls ( $F_{\mathrm{AD} \text { vs. controls }}(1,95)=0.03, p=$ $\left.0.87, r=0.02 ; F_{\mathrm{CD}+\mathrm{AD} \text { vs. controls }}(1,95)=0.63, p=0.43, r=0.08\right)$, whereas $\mathrm{CD}$-only participants performed worse than controls $(F(1,95)=6.78, p=0.01, r=0.26)$. The three-way $\mathrm{CD} \times \mathrm{AD} \times$ emotion interaction was non-significant $(F(4,380)=1.31, p=0.26)$. However, Figure 2 illustrates a degree of unevenness in the effect across emotions, with the largest CD-related deficits observed for fear. 
[Figure 2 about here]

There was a significant $\mathrm{AD} \times$ emotion $\mathrm{x}$ morph interaction $(F(6,596)=2.71, p=0.01)$. For happiness, disgust and sadness, $A D+$ individuals performed better than $A D$ - individuals but only for the lowest intensity emotions (all $p<0.05$ ). Conversely, for low-intensity anger, $A D+$ individuals performed worse than AD- individuals $(p<0.01)$; see Figure S3.

When collapsing across the CD-only and comorbid CD+ADs groups, $C U+$ individuals performed worse than $\mathrm{CU}$ - individuals for fear, happiness and disgust recognition (CU traits $\mathrm{x}$ emotion: $F(3,107)$ $=5.28, p<0.01)$. However, $\mathrm{CU}$ traits did not interact with fixation position (see Figure S4).

\section{Response biases}

Overall, participants were most likely to label neutral faces as sad (see Figure 3). Individuals without ADs were more likely to classify neutral faces as angry than those with $\operatorname{ADs}(F(1,95)=5.48, p=$ $0.02, r=0.23)$. CD participants were more likely to classify neutral faces as fearful $(F(1,95)=8.30, p$ $<0.01, r=0.28)$, and less likely than those without CD to classify neutral faces as sad $(F(1,95)=$ 4.56, $p=0.035, r=0.21)$. Individuals with ADs were more likely to classify neutral faces as sad than those without ADs, although this effect was only marginally significant $(F(1,95)=3.52, p=0.06$, $r=0.19)$. There were no significant $C D \times A D$ interactions for response biases for any emotion.

[Figure 3 about here]

Confounding factors

There were group differences in IQ, gender and depressive symptoms. All of the outcome variables that were modulated by $C D$ or $A D$ status, apart from the classification of neutral faces as angry, were significantly correlated with one or more of these confounding variables. IQ was positively correlated with overall emotion recognition, and the tendency to categorise neutral faces as fearful or sad (all $p<$ 0.01). Females (vs. males) performed significantly better on low-intensity happiness, sadness and disgust faces (averaged, $r=0.35, p<0.01$ ), but significantly worse on low-intensity anger $(r=-0.32$, $p<0.01)$, with an increased tendency to categorise neutral faces as sad $(r=0.26, p=0.01)$ and a reduced tendency to categorise them as fearful $(r=-0.22, p=0.03)$. The results of five separate hierarchical regression analyses exploring these relationships further are presented in Table 2.

[Table 2 about here] 
The inclusion of IQ as a predictor reduced the effect of CD on overall emotion recognition, as well as the tendency for CD participants to label neutral faces as fearful or sad. Given that IQ was significantly related to $C D$, the effect of $C D$ on emotion recognition may be partly explained by lower IQ. The inclusion of gender as a predictor also reduced the effect of ADs on the categorisation of neutral faces as sad. However, whilst gender was also a significant predictor of low-intensity emotion recognition performance, this was no longer the case when AD status was added as a predictor.

\section{Discussion}

We investigated sensitivity and bias in emotion recognition in adolescents with $C D, A D s$ and comorbid CD+ADs. Several key findings emerged: First, individuals with CD showed reduced sensitivity to all emotions, irrespective of comorbid ADs, supporting our hypothesis that $C D$ individuals would show generalised emotion recognition impairments. This is consistent with previous studies on emotion recognition in both CD (Fairchild et al., 2010; Fairchild et al., 2009; Sully et al., 2015) and CU traits/psychopathy (Marsh \& Blair, 2008). However, these results may have been driven, at least in part, by group differences in IQ.

Second, participants with ADs showed increased sensitivity to low-intensity sadness, happiness and disgust compared to those without ADs. This partially supports previous research in which anxious adults were shown to be hypersensitive to ambiguous stimuli (e.g. Richards et al., 2002). However, participants with ADs showed decreased sensitivity to low-intensity anger compared to those without ADs. This was unexpected, given that anxious individuals are considered to be hypersensitive to threat (e.g. Bar-Haim et al., 2007). Our regression analyses suggested that these effects were not modulated by gender.

Third, our manipulation of fixation position was successful: mouth-region fixation generally resulted in poorer performance, and fear was most easily recognised when fixating the eyes, as expected. However, there were no significant interactions between fixation and either CD status or CU traits; deficits in fear recognition in the CD group (across high and low CU traits) were not ameliorated when participants fixated the eyes. Importantly, this runs counter to Dadds et al. (2006), where CU traits were associated with fear recognition deficits, except when individuals were instructed to fixate the eye region of the face. This may be due to our CD sample not showing the full range of scores on the 
ICU; however, we did find that CU traits were related to impaired fear, happiness and sadness recognition, consistent with previous research (e.g. Blair \& Coles, 2000).

Fourth, the comorbid $C D+A D s$ group did not significantly differ from controls on any emotion. This suggests that the AD-related emotion processing style (heightened sensitivity to threat) may counteract the impairments associated with CD. It also suggests that antisocial behaviour in this group may result from factors other than emotion recognition deficits (e.g., emotional dysregulation, see Frick \& Morris, 2004). However, longitudinal studies are needed to test this hypothesis. Further work should ascertain the extent to which the apparent protective effect of comorbid ADs is explained by an increased proportion of females and higher IQ (albeit non-significantly so) in the comorbid versus the pure CD group.

Fifth, group effects on response bias were less marked than sensitivity effects. All participants, regardless of $C D$ or $A D$ status, were most likely to label neutral faces as sad compared to the other emotions, consistent with previous research with healthy adults (e.g. Gur et al., 2002). In terms of psychopathology, individuals with CD were less likely to label neutral faces as sad, consistent with previous findings in adults (Hoaken, Allaby, \& Earle, 2007). In addition, CD individuals (collapsed across $A D$ status) were more likely to classify neutral faces as fearful. This was unexpected, given the well-documented relationship between antisocial/aggressive behaviour and hostile attributional biases (e.g. Dodge, Price, Bachorowski, \& Newman, 1990; Lochman \& Dodge, 1994), and contradicted our initial hypotheses regarding response bias. However, very few studies have examined attributional biases via classification of neutral faces and results have been mixed: one study found that violent offenders were more likely to label neutral faces as disgusted compared to non-violent offenders (Hoaken et al., 2007), whereas another found that violent offenders were more likely than controls to label ambiguous faces as angry (Schönenberg \& Jusyte, 2014). It is also important to note that the observed group differences in response bias may be related to differences in IQ. Contrary to theories relating anxiety to threat biases (e.g. Beck \& Clark, 1997), individuals with ADs were less likely to label neutral faces as angry, and more likely to label them as sad, compared to individuals without ADs, although the latter effect may have been driven by group differences in gender. 


\section{Limitations}

The results of the present study should be interpreted in the light of several limitations. First, group differences in IQ may have been responsible for the emotion recognition deficits seen in CD. However, many studies have found that CD is associated with lower IQ (Farrington, 1995; Frick et al., 1991; Lynam, Moffitt, \& Stouthamer-Loeber, 1993; Moffitt, Gabrieli, Mednick, \& Schulsinger, 1981; Moffitt \& Silva, 1988), suggesting that our CD group is broadly representative of the CD population, and previous studies with IQ-matched controls also observed deficits in emotion recognition in CD (Fairchild et al., 2009). Whilst future studies may address this issue by matching groups on IQ, caution should be taken to avoid reducing the construct validity of $C D$ by recruiting higher IQ CD participants. Second, our sample size was not sufficiently large to detect subtle differences in emotion recognition between the $C D$ and comorbid $C D+A D$ groups. This is, nevertheless, the largest study of its kind and provides a foundation for future research. Third, three individuals in our CD groups had ODD plus sub-threshold CD. There is considerable comorbidity (as well as DSM-IV symptom overlap) between these disorders (see Maughan, Rowe, Messer, Goodman, \& Meltzer, 2004), therefore it would be very difficult to identify "pure" cases of CD. Importantly, exclusion of ODD-only individuals did not affect the results. Fourth, it is possible that our method for ascertaining a research diagnosis of $C D$ and ADs may have led to symptom inflation. However, the K-SADS-PL was used only to assign participants to groups, and most of our CD and AD participants had more symptoms than required to meet diagnostic criteria. Fifth, depression was common among our clinical groups. It is possible that depression and anxiety differentially impact emotion recognition (e.g. Demenescu, Kortekaas, den Boer, \& Aleman, 2010), however, neither the level of depressive symptoms nor the rate of MDD diagnoses differed among our clinical groups and depressive symptoms did not correlate with emotion recognition.

\section{Conclusions}

This is the first study to investigate emotion recognition in individuals with $C D$ and ADs using a sensitive task involving morphed facial expressions and a fixation location manipulation. CD was associated with impaired recognition of emotional expressions, although these effects appear to be related to lower IQ. In addition, ADs were associated with increased sensitivity to low-intensity happiness, disgust and sadness, which was not accounted for by group differences in gender. The 
comorbid $C D+A D$ group did not differ from controls in recognition of any of the emotions, suggesting that comorbid ADs may counteract the effects on $C D$ on emotion recognition. 
Acknowledgements

The research was supported by a Vice Chancellor scholarship from the University of Southampton to R.S. and an Adventure in Research grant awarded to G.F.

We thank our participants and their parents for taking part in the study, and the Youth Offending Teams, Pupil Referral Units, schools and colleges for their help with recruitment. We acknowledge the contribution of Alex James, Lara Barbuselu, Emily Warr, Kate Sully, Nadia Peppa, Vicky Rothery and Georgie Lithauer to data collection. 
- Both conduct disorder (CD) and anxiety disorders (ADs) are associated with altered emotion recognition.

- These disorders frequently co-occur, yet little is known about emotion recognition in individuals with comorbid CD+ADs.

- We investigated facial emotion recognition in adolescents with $C D, A D s$, comorbid CD+ADs and controls, using a task involving morphed facial expressions.

- $\quad C D$ alone was associated with general emotion recognition impairments, whereas ADs and comorbid CD+ADs were associated with normal or enhanced performance.

- The presence of comorbid ADs appeared to counteract the effects of CD on facial emotion recognition.

- Our findings have implications for targeted interventions: e.g., emotion training interventions may be more effective in individuals with $C D$ alone than those with comorbid $A D s$. 


\section{References}

Adams, W.J., Gray, K.L., Garner, M., \& Graf, E.W. (2010). High-level face adaptation without awareness. Psychological Science, 21(2), 205-210.

American Psychiatric Association. (2013). Diagnostic and Statistical Manual of Mental Disorders (5th edn.). Washington, D.C.: Author.

Angold, A., Costello, E.J., \& Erkanli, A. (1999). Comorbidity. Journal of Child Psychology and Psychiatry, 40(1), 57-87.

Bar-Haim, Y., Lamy, D., Pergamin, L., Bakermans-Kranenburg, M.J., \& van IJzendoorn, M.H. (2007). Threat-related attentional bias in anxious and nonanxious individuals: a meta-analytic study. Psychological Bulletin, 133(1), 1-24.

Battaglia, M., Zanoni, A., Ogliari, A., Crevani, F., Falzone, L., Bertoletti, E., \& Di Serio, C. (2010). Identification of gradually changing emotional expressions in schoolchildren: the influence of the type of stimuli and of specific symptoms of anxiety. Cognition \& Emotion, 24(6), 10701079.

Beck, A.T., \& Clark, D.A. (1997). An information processing model of anxiety: automatic and strategic processes. Behaviour Research and Therapy, 35(1), 49-58.

Benton, A.L., Hamsher, K.d.S., Varney, N.R., \& Spreen, O. (1994). Contributions to neuropsychological assessment: a clinical manual. New York, NY: Oxford University Press.

Blair, R.J.R., \& Coles, M. (2000). Expression recognition and behavioural problems in early adolescence. Cognitive Development, 15, 421-434.

Cohen, J. (1992). A power primer. Psychological Bulletin, 112(1), 155-159.

Crick, N.R., \& Dodge, K.A. (1994). A review and reformulation of social information-processing mechanisms in children's social adjustment. Psychological Bulletin, 115(1), 74-101.

Crum, R.M., Lillie-Blanton, M., \& Anthony, J.C. (1996). Neighborhood environment and opportunity to use cocaine and other drugs in late childhood and early adolescence. Drug and Alcohol Dependence, 43(3), 155-161.

Dadds, M.R., El Masry, Y., Wimalaweera, S., \& Guastella, A.J. (2008). Reduced eye gaze explains "fear blindness" in childhood psychopathic traits. Journal of the American Academy of Child and Adolescent Psychiatry, 47(4), 455-463.

Dadds, M.R., Perry, Y., Hawes, D.J., Merz, S., Riddell, A.C., Haines, D.J., Solak, E., \& Abeygunwardane, A.I. (2006). Attention to the eyes and fear-recognition deficits in child psychopathy. British Journal of Psychiatry, 189, 280-281.

Demenescu, L.R., Kortekaas, R., den Boer, J.A., \& Aleman, A. (2010). Impaired attribution of emotion to facial expressions in anxiety and major depression. PLoS One, 5(12), e15058.

Dodge, K.A., Price, J.M., Bachorowski, J., \& Newman, J.P. (1990). Hostile attributional biases in severely aggressive adolescents. Journal of Abnormal Psychology, 99(4), 385-392.

Fairchild, G., Stobbe, Y., van Goozen, S.H., Calder, A.J., \& Goodyer, I.M. (2010). Facial expression recognition, fear conditioning, and startle modulation in female subjects with conduct disorder. Biological Psychiatry, 68(3), 272-279.

Fairchild, G., Van Goozen, S.H., Calder, A.J., Stollery, S.J., \& Goodyer, I.M. (2009). Deficits in facial expression recognition in male adolescents with early-onset or adolescence-onset conduct disorder. Journal of Child Psychology and Psychiatry, 50(5), 627-636.

Farrington, D.P. (1995). The development of offending and antisocial behaviour from childhood: Key findings from the Cambridge Study in Delinquent Development. Journal of Child Psychology and Psychiatry, 36(6), 929-964.

Frick, P.J. (2004). The Inventory of Callous-Unemotional Traits. Unpublished rating scale. The University of New Orleans.

Frick, P.J., Kamphaus, R.W., Lahey, B.B., Loeber, R., Christ, M.G., Hart, E.L., \& Tannenbaum, L. (1991). Academic underachievement and the disruptive behavior disorders. Journal of Consulting and Clinical Psychology, 59(2), 289-294.

Frick, P.J., Lilienfeld, S.O., Ellis, M., Loney, B., \& Silverthorn, P. (1999). The association between anxiety and psychopathy dimensions in children. Journal of Abnormal Child Psychology, 27(5), 383-392.

Frick, P.J., \& Morris, A.S. (2004). Temperament and developmental pathways to conduct problems. Journal of Clinical Child and Adolescent Psychology, 33(1), 54-68.

Greene, R.W., Biederman, J., Zerwas, S., Monuteaux, M.C., Goring, J.C., \& Faraone, S.V. (2002). Psychiatric comorbidity, family dysfunction, and social impairment in referred youth with oppositional defiant disorder. American Journal of Psychiatry, 159, 1214-1224. 
Gur, R.C., Sara, R., Hagendoorn, M., Marom, O., Hughett, P., Macy, L., Turner, T., Bajcsy, R., Posner, A., \& Gur, R.E. (2002). A method for obtaining 3-dimensional facial expressions and its standardixation for use in neurocognitive studies. Journal of Neuroscience Methods, 115, 137-143.

Guyer, A.E., McClure, E.B., Adler, A.D., Brotman, M.A., Rich, B.A., Kimes, A.S., Pine, D.S., Ernst, M., \& Leibenluft, E. (2007). Specificity of facial expression labeling deficits in childhood psychopathology. Journal of Child Psychology and Psychiatry, 48(9), 863-871.

Hoaken, P.N., Allaby, D.B., \& Earle, J. (2007). Executive cognitive functioning and the recognition of facial expressions of emotion in incarcerated violent offenders, non-violent offenders, and controls. Aggressive Behavior, 33(5), 412-421.

Ialongo, N., Edelsohn, G., Werthamer-Larsson, L., Crockett, L.J., \& Kellam, S. (1996). The course of aggression in first-grade children with and without comorbid anxious symptoms. Journal of Abnormal Child Psychology, 24(4), 445-456.

Jarros, R.B., Salum, G.A., Belem da Silva, C.T., Toazza, R., de Abreu Costa, M., Fumagalli de Salles, J., \& Manfro, G.G. (2012). Anxiety disorders in adolescence are associated with impaired facial expression recognition to negative valence. Journal of Psychiatry Research, 46(2), 147151.

Kaufman, J., Birmaher, B., Brent, D., Rao, U., Flynn, C., Moreci, P., Williamson, D., \& Ryan, N.D. (1997). Schedule for affective disorders and schizophrenia for school-age children - present and lifetime version (K-SADS-PL): initial reliability and validity data. Journal of the American Academy of Child \& Adolescent Psychiatry, 36, 980-988.

Kendall, P.C., Brady, E.U., \& Verduin, T.L. (2001). Comorbidity in childhood anxiety disorders and treatment outcome. Journal of the American Academy of Child \& Adolescent Psychiatry, 40(7), 787-794.

Lahey, B.B., Loeber, R., Burke, J., Rathouz, P.J., \& McBurnett, K. (2002). Waxing and waning in concert: dynamic comorbidity of conduct disorder with other disruptive and emotional problems over 17 years among clinic-referred boys. Journal of Abnormal Psychology, 111(4), 556-567.

Leist, T., \& Dadds, M.R. (2009). Adolescents' ability to read different emotional faces relates to their history of maltreatment and type of psychopathology. Clinical Child Psychology and Psychiatry, 14(2), 237-250.

Lochman, J.E., \& Dodge, K.A. (1994). Social-cognitive processes of severely violent, moderately aggressive and nonaggressive boys. Journal of Consulting and Clinical Psychology, 62(2), 366-374.

Lynam, D.R., Moffitt, T.E., \& Stouthamer-Loeber, M. (1993). Explaining the relation between IQ and delinquency: class, race, test motivation, school failure, or self-control? Journal of Abnormal Psychology, 102(2), 187-196.

Manassis, K., \& Young, A. (2000). Perception of emotions in anxious and learning disabled children. Depression and Anxiety, 12, 209-216.

Marsh, A.A., \& Blair, R.J.R. (2008). Deficits in facial affect recognition among antisocial populations: a meta-analysis. Neuroscience and Behavioral Reviews, 32(3), 454-465.

Maughan, B., Rowe, R., Messer, J., Goodman, R., \& Meltzer, H. (2004). Conduct disorder and oppositional defiant disorder in a national sample: developmental epidemiology. Journal of Child Psychology and Psychiatry, 45(3), 609-621.

McClure, E.B., Pope, K., Hoberman, A.J., Pine, D.S., \& Leibenluft, E. (2003). Facial expression recognition in adolescents with mood and anxiety disorders. American Journal of Psychiatry, $160,1172-1174$.

Melfsen, S., \& Florin, I. (2002). Do socially anxious children show deficits in classifying facial expressions of emotions? Journal of Nonverbal Behavior, 26(2), 109-126.

Miller, J. (1996). The sampling distribution of $d$ '. Perception and Psychophysics, 58(1), 65-72.

Moffitt, T.E., Gabrieli, W.F., Mednick, S.A., \& Schulsinger, F. (1981). Socioeconomic status, IQ and delinquency. Journal of Abnormal Psychology, 90(2), 152-156.

Moffitt, T.E., \& Silva, P.A. (1988). IQ and delinquency: a direct test of the differential detection hypothesis. Journal of Abnormal Psychology, 97(3), 330-333.

Pajer, K., Leininger, L., \& Gardner, W. (2010). Recognition of facial affect in girls with conduct disorder. Psychiatry Research, 175(3), 244-251.

Polier, G.G., Vloet, T.D., Herpertz-Dahlmann, B., Laurens, K.R., \& Hodgins, S. (2012). Comorbidity of conduct disorder symptoms and internalising problems in children: investigating a community and a clinical sample. European Child and Adolescent Psychiatry, 21(1), 31-38. 
Rayner, K. (1998). Eye movements in reading and information processing: 20 years of research. Psychological Bulletin, 124(3), 372-422.

Reeb-Sutherland, B.C., Rankin Williams, L., Degnan, K.A., Perez-Edgar, K., Chronis-Tuscano, A., Leibenluft, E., Pine, D.S., Pollak, S.D., \& Fox, N.A. (2015). Identification of emotional facial expressions among behaviorally inhibited adolescents with lifetime anxiety disorders. Cognition and Emotion, 29(2), 372-382.

Richards, A., French, C.C., Calder, A.J., Webb, B., Fox, R., \& Young, A.W. (2002). Anxiety-related bias in the classification of emotionally ambiguous facial expressions. Emotion, 2(3), 273-287.

Robins, L.N. (1978). Sturdy childhood predictors of adult antisocial behaviour: replications from longitudinal studies. Psychological Medicine, 8(04), 611-622.

Schepman, K., Taylor, E., Collishaw, S., \& Fombonne, E. (2012). Face emotion processing in depressed children and adolescents with and without comorbid conduct disorder. Journal of Abnormal Child Psychology, 40(4), 583-593.

Schönenberg, M., \& Jusyte, A. (2014). Investigation of the hostile attribution bias toward ambiguous facial cues in antisocial violent offenders. European Archives of Psychiatry and Clinical Neuroscience, 264(1), 61-69.

Scott, S., Henderson, J.M., Knapp, M., \& Maughan, B. (2001). Financial cost of social exclusion: follow up study of antisocial children into adulthood. The BMJ, 323, 1-5.

Simonian, S.J., Beidel, D.C., Turner, S.M., Berkes, J.L., \& Long, J.H. (2001). Recognition of facial affecy by children and adolescents diagnosed with social phobia. Child Psychiatry and Human Development, 32(2), 137-145.

Sourander, A., Jensen, P., Davies, M., Niemela, S., Elonheimo, H., Ristkari, T., Helenius, H., Sillanmaki, L., Piha, J., Kumpulainen, K., Tamminen, T., Moilanen, I., \& Almqvist, F. (2007). Who is at greatest risk of adverse long-term outcomes? The Finnish from a boy to a man study. Journal of the American Academy of Child \& Adolescent Psychiatry, 46(9), 1148-1161.

Spielberger, C.D., Gorsuch, R.L., Lushene, R., Vagg, P.R., \& Jacobs, G.A. (1983). Manual for the State-Trait Anxiety Inventory. Palo Alto, CA: Consulting Psychologists Press.

Sully, K., Sonuga-Barke, E.J., \& Fairchild, G. (2015). The familial basis of facial emotion recognition deficits in adolescents with conduct disorder and their unaffected relatives. Psychological Medicine, 1-11.

The Mathworks. (2012). MATLAB and statistics toolbox 8.1. Natik, Massachusetts: The Mathworks, Inc.

Tottenham, N., Tanaka, J.W., Leon, A.C., McCarry, T., Nurse, M., Hare, T.A., Marcus, D.J., Westerlund, A., Casey, B.J., \& Nelson, C. (2009). The NimStim set of facial expressions: judgments from untrained research participants. Psychiatry Research, 168(3), 242-249.

Walker, J.L., Lahey, B.B., Russo, M.F., Frick, P.J., Christ, M.G., McBurnett, K., Loeber, R., Stouthamer-Loeber, M., \& Green, S.M. (1991). Anxiety, inhibition and conduct disorder in children: I. Relations to social impairment. Journal of the American Academy of Child \& Adolescent Psychiatry, 30(2), 187-191.

Wechsler, D. (1999). Wechsler Abbreviated Scale of Intelligence (WASI). San Antonio, TX: The Psychological Corporation, Harcourt Brace and Company.

Zigmond, A.S., \& Snaith, R.P. (1983). The Hospital Anxiety and Depression Scale. Acta Psychiatrica Scandinavica, 67(6), 361-370. 
Table 1: Participant characteristics

\begin{tabular}{|c|c|c|c|c|c|c|c|c|c|}
\hline & \multicolumn{2}{|c|}{$\begin{array}{c}\text { Control }^{1} \\
\mathrm{~N}=28,22 \\
\text { males }\end{array}$} & \multicolumn{2}{|c|}{$\begin{array}{c}\mathrm{CD}^{2} \\
\mathrm{~N}=28,23 \\
\text { males }\end{array}$} & \multicolumn{2}{|c|}{$\begin{array}{c}\text { Anxious }^{3} \\
\mathrm{~N}=23,5 \\
\text { males }\end{array}$} & \multicolumn{2}{|c|}{$\begin{array}{c}\text { Comorbid } \\
\mathrm{N}=20,12 \\
\text { males }\end{array}$} & \multirow[t]{2}{*}{$F$} \\
\hline & Mean & SD & Mean & SD & Mean & SD & Mean & SD & \\
\hline Age (years) & 16.20 & 1.47 & 16.74 & 1.49 & 16.42 & 1.93 & 16.84 & 1.08 & 0.92 \\
\hline Intelligence Quotient & 109.69 & 11.92 & 95.59 & 9.23 & 102.78 & 11.44 & 103.15 & 13.16 & $7.12^{\star \star}$ \\
\hline Neighbourhood (NES) & 4.79 & 2.65 & 7.59 & 5.12 & 6.52 & 3.46 & 6.75 & 3.82 & 2.59 \\
\hline Face identity (BFRT) & 44.83 & 5.56 & 45.52 & 3.72 & 47.39 & 3.92 & 49.55 & 11.60 & 2.39 \\
\hline CD Symptoms $^{\dagger}$ & 0.10 & 0.41 & 8.04 & 3.16 & 0.24 & 0.62 & 7.43 & 3.18 & $\begin{array}{c}13.40^{* *} \\
2,4>1,3\end{array}$ \\
\hline CU traits & 20.07 & 6.69 & 30.02 & 9.94 & 19.02 & 6.98 & 27.23 & 9.72 & $\begin{array}{c}10.18^{\star *} \\
2,4>1,3\end{array}$ \\
\hline STAI Trait Anxiety & 36.10 & 9.87 & 43.33 & 10.94 & 51.93 & 10.46 & 57.35 & 9.66 & $\begin{array}{c}20.28^{* *} \\
3,4>1,2\end{array}$ \\
\hline HAD Depression & 3.52 & 2.90 & 7.81 & 4.29 & 5.78 & 4.02 & 7.25 & 4.15 & $\begin{array}{c}6.72^{* *} \\
2,4>1\end{array}$ \\
\hline \multicolumn{10}{|l|}{$\begin{array}{l}\text { Psychotropic } \\
\text { medication - } n \text { (\%) }\end{array}$} \\
\hline Antidepressants & \multicolumn{2}{|c|}{0} & \multicolumn{2}{|c|}{$1(3.6)$} & \multicolumn{2}{|c|}{$5(21.7)$} & \multicolumn{2}{|c|}{$2(10.0)$} & \\
\hline Stimulants (for ADHD) & \multicolumn{2}{|c|}{0} & \multicolumn{2}{|c|}{$2(7.1)$} & \multicolumn{2}{|c|}{0} & \multicolumn{2}{|c|}{0} & \\
\hline \multicolumn{10}{|c|}{${ }^{\star \star} p<0.01,{ }^{\dagger}$ Number of lifetime conduct disorder (CD) symptoms endorsed (out of 13). } \\
\hline \multicolumn{10}{|c|}{ ADHD=Attention-deficit/hyperactivity disorder, BFRT=Benton Face Recognition Test, } \\
\hline \multicolumn{10}{|c|}{$\mathrm{CU}=$ callous-unemotional, $\mathrm{HAD}=$ Hospital Anxiety and Depression scales, NES=Neighbourhood } \\
\hline & 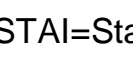 & & & & & & & & \\
\hline
\end{tabular}


Table 2 Results of the hierarchical regression analyses

\begin{tabular}{|c|c|c|c|c|c|c|c|c|c|c|}
\hline & \multicolumn{6}{|c|}{ Low-intensity } & & & & \\
\hline & \multirow{2}{*}{\multicolumn{2}{|c|}{$\begin{array}{l}\text { Recognition } \\
\text { accuracy (d') }\end{array}$}} & \multirow{2}{*}{\multicolumn{2}{|c|}{$\begin{array}{c}\text { happy, sad \& } \\
\text { disgust }^{\mathrm{a}}\end{array}$}} & \multirow{2}{*}{\multicolumn{2}{|c|}{$\begin{array}{l}\text { Low-intensity } \\
\text { anger }{ }^{\mathrm{b}}\end{array}$}} & \multirow{2}{*}{\multicolumn{2}{|c|}{ Neutral as fear }} & \multirow{2}{*}{\multicolumn{2}{|c|}{ Neutral as sad }} \\
\hline & & & & & & & & & & \\
\hline & Beta $^{\dagger}$ & $\mathrm{R}^{2}$ & Beta & $\mathrm{R}^{2}$ & Beta & $\mathrm{R}^{2}$ & Beta & $\mathrm{R}^{2}$ & Beta & $\mathrm{R}^{2}$ \\
\hline Step 1 & & $0.15^{\star *}$ & & $0.15^{\star *}$ & & $0.12^{* *}$ & & $0.19^{* *}$ & & $0.14^{\star \star}$ \\
\hline IQ & $0.39^{* *}$ & & 0.17 & & -0.10 & & $-0.38^{* *}$ & & $0.28^{\star *}$ & \\
\hline Gender & - & & $0.37^{* *}$ & & $-0.34^{\star *}$ & & $-0.26^{\star *}$ & & $0.29^{\star *}$ & \\
\hline Step 2 & & $0.19^{* *}$ & & $0.21^{\star *}$ & & $0.17^{* *}$ & & $0.20^{* *}$ & & $0.16^{* *}$ \\
\hline $\mathrm{IQ}$ & $0.35^{* *}$ & & 0.14 & & -0.06 & & $-0.33^{* *}$ & & $0.24^{*}$ & \\
\hline Gender & - & & $0.24^{*}$ & & -0.20 & & $-0.23^{*}$ & & $0.23^{*}$ & \\
\hline CD & -0.13 & & -0.35 & & 0.94 & & 0.15 & & -0.09 & \\
\hline$A D$ & 0.13 & & $0.28^{\star *}$ & & $-0.26^{*}$ & & 0.01 & & 0.09 & \\
\hline Step 3 & & $0.19^{\star *}$ & & $0.21^{* *}$ & & $0.18^{* *}$ & & $0.21^{* *}$ & & $0.16^{* *}$ \\
\hline IQ & $0.35^{\star *}$ & & 0.13 & & -0.05 & & $-0.32^{\star *}$ & & $0.25^{*}$ & \\
\hline Gender & - & & $0.25^{\star}$ & & -0.21 & & $-0.23^{*}$ & & $0.23^{*}$ & \\
\hline CD & -0.13 & & -0.03 & & -0.96 & & 0.15 & & -0.09 & \\
\hline$A D$ & 0.13 & & $0.27^{\star \star}$ & & $-0.26^{*}$ & & 0.01 & & 0.09 & \\
\hline$C D^{*} A D$ & 0.01 & & 0.03 & & -0.03 & & -0.01 & & -0.03 & \\
\hline
\end{tabular}



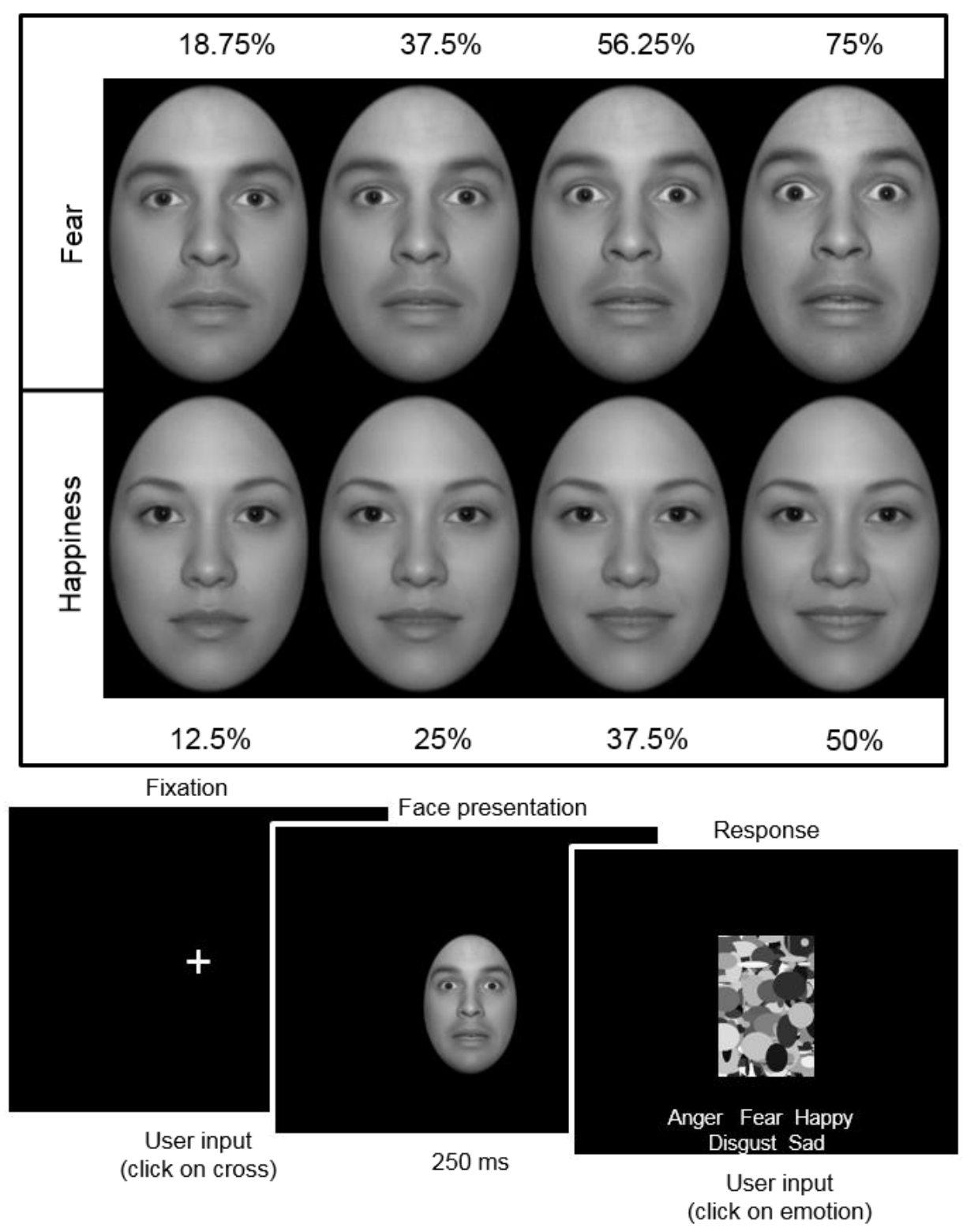

Figure 1: Example stimuli (top) and a schematic representation of a trial (bottom), showing a fearful male face presented at the eye-fixation position, followed by a visual mask. 


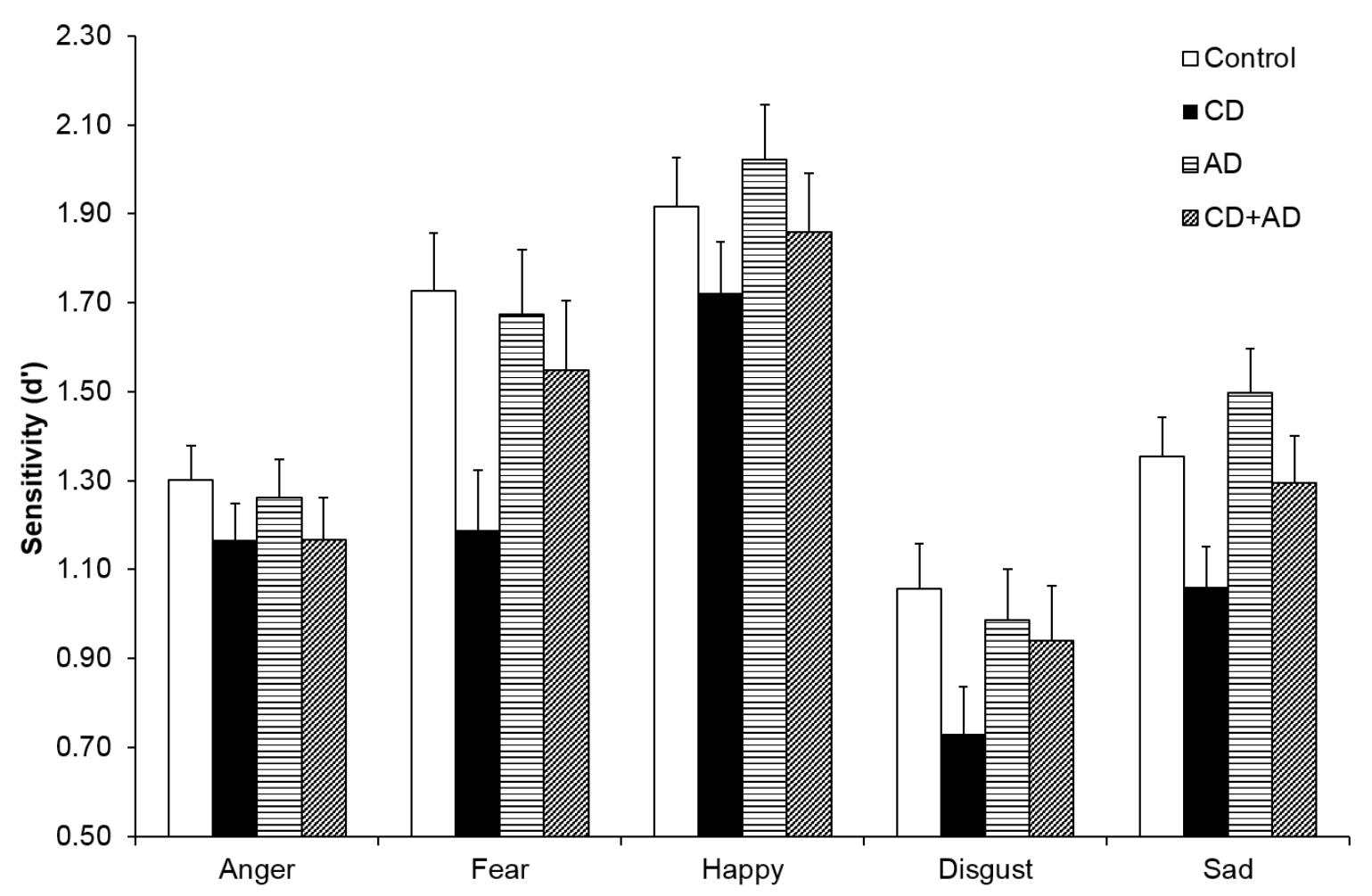

Figure 2: Effect of group on emotion recognition sensitivity, averaged across fixation position and morph strength, and expressed as d-prime ( $\left.d^{\prime}\right)$. Error bars represent \pm 1 S.E.M. AD=anxiety disorder, $\mathrm{CD}=$ conduct disorder. 


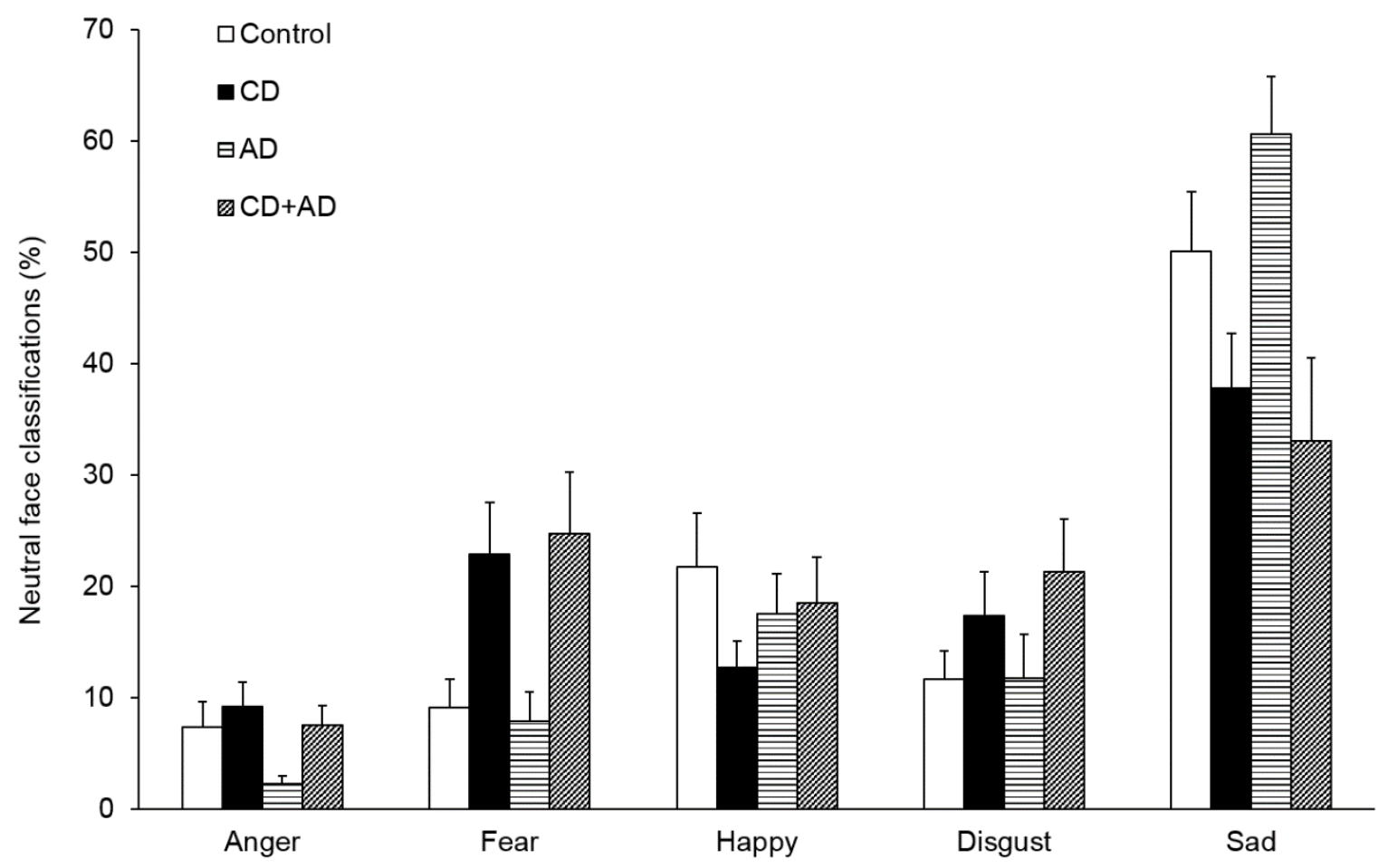

Figure 3: The effects of conduct disorder (CD) and anxiety disorder (AD) on the classification of neutral faces as emotional (error bars represent \pm 1 S.E.M.). 